\title{
Intraventricular Hemorrhage in Premature Infants: Mechanism of Disease
}

\author{
PRAVEEN BALLABH \\ Department of Pediatrics, Anatomy and Cell Biology, New York Medical College-Westchester Medical Center, Valhalla, New York 10595
}

\begin{abstract}
Intraventricular hemorrhage (IVH) is a major complication of prematurity. IVH typically initiates in the germinal matrix, which is a richly vascularized collection of neuronal-glial precursor cells in the developing brain. The etiology of IVH is multifactorial and is primarily attributed to the intrinsic fragility of the germinal matrix vasculature and the disturbance in the cerebral blood flow (CBF). Although this review broadly describes the pathogenesis of $\mathrm{IVH}$, the main focus is on the recent development in molecular mechanisms that elucidates the fragility of the germinal matrix vasculature. The microvasculature of the germinal matrix is frail because of an abundance of angiogenic blood vessels that exhibit paucity of pericytes, immaturity of basal lamina, and deficiency of glial fibrillary acidic protein (GFAP) in the ensheathing astrocytes endfeet. High VEGF and angiopoietin-2 levels activate a rapid angiogenesis in the germinal matrix. The elevation of these growth factors may be ascribed to a relative hypoxia of the germinal matrix perhaps resulting from high metabolic activity and oxygen consumption of the neural progenitor cells. Hence, the rapid stabilization of the angiogenic vessels and the restoration of normal CBF on the first day of life are potential strategies to prevent IVH in premature infants. (Pediatr Res 67: 1-8, 2010)
\end{abstract}

$\mathrm{A}$ pproximately 12,000 premature infants develop intraventricular hemorrhage (IVH) every year in the United States alone $(1,2)$. The incidence of IVH in very low birth weight (VLBW) infants $(<1500 \mathrm{~g})$ has declined from 40 to $50 \%$ in the early 1980 s to $20 \%$ in the late 1980 s (3). However, in the last two decades the occurrence of IVH has remained stationary (4). In extremely premature infants weighing 500$750 \mathrm{~g}$, IVH occurs in about $45 \%$ of neonates (5). Thus, IVH continues to be a major problem of premature infant in modern neonatal intensive care units (NICUs) worldwide.

IVH characteristically initiates in the periventricular germinal matrix. The germinal matrix, located on the head of caudate nucleus and underneath ventricular ependyma, is a highly vascular collection of glial and neuronal precursor cells (Fig. $1 A$ and $B$ ). This periventricular region is selectively vulnerable to hemorrhage in premature infants predominantly in the first $48 \mathrm{~h}$ of life. When the hemorrhage in the germinal

Received July 30, 2009; accepted September 15, 2009.

Correspondence: Praveen Ballabh, M.D., Regional Neonatal Center, 2nd Floor, Maria Fareri Children's Hospital at Westchester Medical Center, Valhalla, NY 10595; e-mail: Pballabh@msn.com

Supported by NIH/NINDS NS050586 grant. matrix is substantial, the ependyma breaks, and the cerebral ventricle fills up with blood. Thus, IVH is typically a progression of germinal matrix hemorrhage. A majority of these infants are asymptomatic and the diagnosis is based on screening cranial ultrasound. Some infants manifest with subtle abnormalities in the level of consciousness, movement, tone, respiration and eye movement; and uncommonly, there is a catastrophic deterioration presenting with stupor, coma, decerebrate posturing, generalized tonic seizure, and quadriparesis.

IVH reduces the survival of premature infants and enhances the risk of a number of neurologic sequelae. A higher mortality rate in premature infants with severe IVH has been reported compared with infants without IVH (6). Premature infants with moderate-to-severe IVH (grade 3-4) are at high risk of posthemorrhagic hydrocephalus, cerebral palsy, and mental retardation, whereas infants with mild IVH (grade 1-2) are at risk of developmental disabilities $(7,8)$. Approximately $45-85 \%$ of premature infants with moderate-to-severe IVH develop major cognitive deficits and about $75 \%$ of these infants need special education in school (9). A recent study has shown better functional outcome of surviving preterm infants with periventricular hemorrhagic infarction at school age than previously thought (10).

Pathogenesis of IVH is multifactorial and is primarily ascribed to a) inherent fragility of the germinal matrix vasculature, $b$ ) disturbance in the cerebral blood flow (CBF), and c) platelet and coagulation disorders. A number of risk factors including vaginal delivery, low Apgar score, severe respiratory distress syndrome, pneumothorax, hypoxia, hypercapnia, seizures, patent ductus arteriosus (PDA), thrombocytopenia, infection, and others predispose to the development of IVH (Table 1) (11-14). These risk factors seem to induce IVH primarily by disturbing the CBF. However, thrombocytopenia contributes to IVH by causing hemostatic failure.

\section{Fragility of Germinal Matrix Vasculature}

Premature infants primarily bleed into the germinal matrix and not in the cortical mantle or white matter, suggesting that

\footnotetext{
Abbreviations: CBF, cerebral blood flow; IVH, intraventricular hemorrhage; GFAP, glial fibrillary acidic protein; TGF $\boldsymbol{\beta}$, transforming growth factor $\beta$; S1P1, sphingosine-1-phosphate1
} 

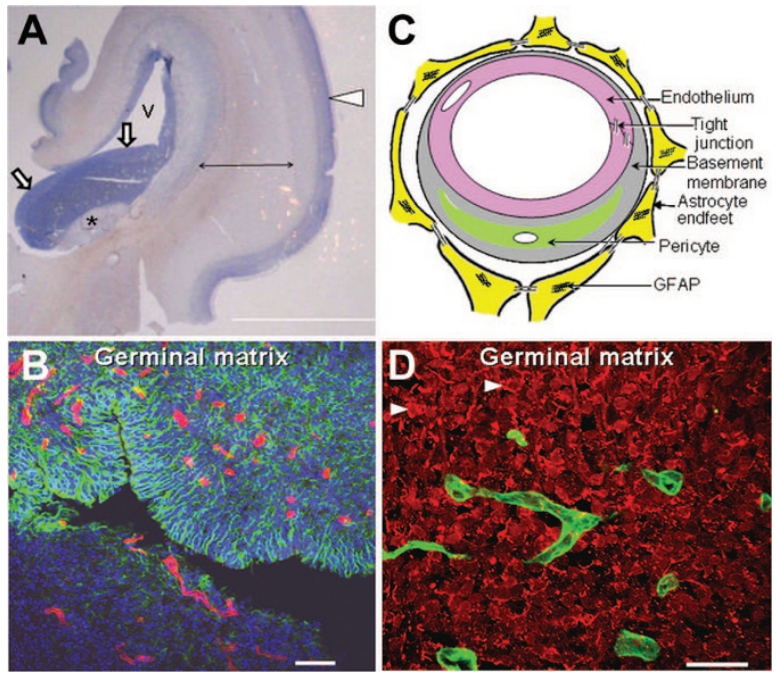

Figure 1. Morphology of the germinal matrix. (A) Representative cresyl violet staining of coronal section of the right-sided cerebral hemisphere of a 20-wk fetus. Note: the cortical plate (arrowhead), white matter (arrow with 2 pointers), germinal matrix (black arrow), caudate nucleus (asterisk), and lateral ventricle (indicated by "v"). The germinal matrix (violet staining) surrounds the whole ventricle but is most conspicuous on the head of caudate nucleus. Scale bar, $0.5 \mathrm{~cm}$. (B) Representative immunofluorescence of cryosection from the germinal matrix of a 24 -wk premature infant labeled with DAPI (blue), GFAP (green), and CD34 (red). Note: the germinal matrix is highly vascular (vascular endothelium in red) and enriched with GFAP (+) glial cells (green). (C) Schematic drawing of the blood-brain barrier in cross-section showing endothelium, endothelial tight junction, basal lamina, pericyte, and astrocyte endfeet. $(D)$ The coronal brain section was double labeled with doublecortin (red, labels neuronal precursors) and CD34 (green, labels endothelium)-specific antibodies. Note: doublecortin (+) neuronal precursor cells are abundantly present in the germinal matrix. Scale bar; 50 (B) and $20 \mu \mathrm{m}(D)$.

there is an intrinsic weakness in the germinal matrix vasculature compared with the other brain regions. Endothelial tight junctions, basement membrane, pericytes, and astrocyte endfeet ensheathing the blood vessels constitute the blood-brain barrier, and immaturity or weakness of any of these components can potentially cause fragility of the germinal matrix vasculature (Fig. 1C). The author's research group has been able to focus on the morphologic and molecular evaluation of germinal matrix vasculature and has unraveled a number of mysteries associated with the components of the blood-brain barrier of this enigmatic brain region (15-20).

Morphology of germinal matrix vasculature. The density and cross-sectional area of the blood vessels are the largest in the human germinal matrix followed by the cerebral cortex and then the white matter for all gestational ages (17-40 wk) (15). In addition, the vascular density and vasculature area in cross-section significantly increases with the advancing gestational age in all the three forebrain regions-germinal matrix, cerebral cortex, and white matter. Interestingly, blood vessels in the germinal matrix are circular in the coronal section, whereas vessels in the cerebral cortex and white matter are relatively flat. The rounded contour of the blood vessel in cross-section indicates immaturity of the germinal matrix vasculature (21). The rich vascularity of the germinal matrix is to meet the high metabolic demand of proliferating and maturing neural precursor cells constituting this brain region. The high vascularity of the germinal matrix enhances the probability of hemorrhage compared with low vascular brain regions; however, this does not entirely explain the mechanism of its intrinsic fragility and propensity to hemorrhage.

Endothelial tight junctions of the germinal matrix. The tight junction is composed of three integral membrane proteins, namely claudin, occludin, and junction adhesion molecules as well as a number of cytoplasmic accessory proteins$\mathrm{ZO} 1, \mathrm{ZO} 2, \mathrm{ZO} 3$, cingulin, and others (22). Tight junction molecules foster cell-to-cell adhesion between endothelial cells and thus contribute to the structural integrity of blood vessels. In addition, tight junction forms a blood-brain barrier to limit the paracellular flux of blood-born substances from entering the brain. The major tight junction proteins, including claudin, occludin, and junction adhesion molecules, have been evaluated in postmortem tissues from human fetuses, preterm, and term infants. Immunohistochemistry and Western blot analysis have shown that the expression of these molecules is similar in the germinal matrix, cerebral cortex, and white matter (16). Thus, endothelial tight junctions are unlikely to be liable for the propensity of germinal matrix vasculature to hemorrhage. Nevertheless, it will be important to evaluate the integrity of signaling events that control the tight junction molecules in the three brain regions. The studies performed on human autopsy material of short postmortem interval are invaluable. However, the limitations of such studies are exposure of premature infants to a number of prenatal and postnatal variables including prenatal steroid, mechanical ventilation, medications and others, which can potentially confound the results.

Perivascular coverage by astrocyte endfeet in the germinal matrix. Astrocyte endfeet ensheath the cerebral vasculature to form a blood-brain barrier and are viewed as providers of structural integrity to the blood vessels. The perivascular coverage by astrocyte endfeet has been evaluated in the forebrain of human fetuses, premature, and mature infants (17). Immunolabeling and analyses of high-resolution images have revealed that perivascular coverage by glial fibrillary acidic protein (GFAP)-positive astrocyte endfeet are lesser in the germinal matrix compared with the cerebral cortex or white matter (17). As GFAP provides shape and mechanical strength to astrocytes, reduced GFAP expression in the astrocytes endfeet might contribute to the fragility of germinal matrix vasculature. Developmentally, GFAP-positive astrocytes are first seen at 9-wk gestational age in the spinal cord, $14 \mathrm{wk}$ in the internal capsule and thalamus, and 14-19 wk in the ependyma of the frontal cortex (23-25). As prenatal glucocorticoids reduce the incidence of IVH, it is important to know how steroids affect the expression of GFAP. There is a lack of in vivo data, however, in vitro studies have shown that glucocorticoids up-regulate GFAP (26). Together, paucity of GFAP in perivascular endfeet of the germinal matrix vasculature might contribute to its fragility and propensity to hemorrhage. It is also likely that prenatal glucocorticoids protect against IVH by up-regulating GFAP in the astrocyte endfeet.

Vascular basal lamina of the germinal matrix is deficient in fibronectin. The basal lamina in the cerebral vasculature is 
Table 1. Neonatal risk factors in the pathogenesis of IVH

\begin{tabular}{|c|c|c|c|}
\hline & Major pathogenic mechanism & Putative mechanisms* & Risk factors \\
\hline \multirow[t]{17}{*}{1 . } & Disturbance in $\mathrm{CBF}$ & Fluctuation in $\mathrm{CBF}$ & Hypoxia \\
\hline & & & Hypercarbia \\
\hline & & & Severe acidosis \\
\hline & & & Asynchrony between infants and ventilator breathe \\
\hline & & & Severe RDS \\
\hline & & & Patent ductus arteriosus \\
\hline & & & Frequent suctioning of airway \\
\hline & & & Rapid infusion of $\mathrm{NaHCO}_{3}$ \\
\hline & & High cerebral venous pressure & Pneumothorax \\
\hline & & & High ventilator pressure \\
\hline & & & Prolonged labor and vaginal delivery \\
\hline & & Abnormal blood pressure & Hypotension \\
\hline & & & Hypertension \\
\hline & & & Sepsis \\
\hline & & & Dehydration \\
\hline & & Pressure-passive circulation & Extreme prematurity and low birth weight $(<1000 \mathrm{~g})$ \\
\hline & & & $\begin{array}{l}\text { Clinically unstable resulting from respiratory compromise, } \\
\text { sepsis, or other reasons }\end{array}$ \\
\hline \multirow[t]{2}{*}{2.} & Inherent fragility of the germinal & Might be worsened by an inflammatory injury to & Hypoxic ischemic insult \\
\hline & matrix vasculature & the blood-brain barrier & Sepsis \\
\hline \multirow[t]{2}{*}{3.} & Platelet and coagulation disturbances & Hemostatic failure & Thrombocytopenia \\
\hline & & & Disseminated intravascular coagulopathy \\
\hline
\end{tabular}

* Correlation of mechanisms with the risk factor is based on available evidence and author's speculations.

composed of laminin, collagen, fibronectin, and heparan sulfate proteoglycan and perlecan $(27,28)$. Mice engineered to eliminate fibronectin, collagen IV, laminin, or perlecan gene have revealed that these molecules are essential for blood vessel formation and its stabilization (29-31). Furthermore, fibronectin promotes cell adhesion, cell migration, and cytoskeletal organization (32). In addition, its polymerization controls the stability of the extracellular matrix by providing tensile and mechanical strength to tissues $(33,34)$.

On the basis of the above premise, a number of investigators have evaluated basal lamina in animal model and postmortem brains from human premature infants. In newborn beagle pups, immunolabeling and confocal microscopy showed that immunoreactivity for laminin and collagen $\mathrm{V}$ in the germinal matrix markedly increased on postnatal $\mathrm{d} 4$ compared with $d$, but not in the white matter (35). A subsequent study from the same group revealed that postnatal indomethacin treatment significantly increased the staining intensity of laminin and collagen $\mathrm{V}$. These studies suggest that laminin and collagen $\mathrm{V}$ deposition with advancing age or indomethacin therapy might stabilize the germinal matrix vasculature (36). Another group of investigators has evaluated collagen I, II, and IV in the germinal matrix compared with the cortex and white matter in immunolabeled human brain sections from fetuses and premature infants (37). They found no difference in the expression of these molecules among the three brain regions.

A comprehensive molecular assessment of the components of basal lamina in autopsy materials from human fetuses and premature infants has shown significantly lower fibronectin protein levels in the germinal matrix relative to the cortical mantle or white matter (18). In contrast, laminin $(\alpha 1, \alpha 4$, and $\alpha 5$ ), perlecan, $\alpha 1$, and $\alpha 4$ (IV) collagen levels are comparable in the three brain regions (18). In addition, low-dose prenatal betamethasone treatment increases fibronectin expression
(18). Because polymerization of fibronectin into the extracellular matrix stabilizes vasculature and fibronectin-null mice display cerebral hemorrhage with embryonic death, diminished expression of fibronectin in the germinal matrix may contribute to the selective fragility of the blood vessels in this brain region. Thus, discovering a novel therapy to up-regulate fibronectin in the germinal matrix vasculature and its validation in animal model might hold promise in the prevention of IVH.

Importantly, both TGF $\beta$ and fibronectin levels are lesser in the germinal matrix compared with the other brain regions. As elevated TGF $\beta$ up-regulates fibronectin in addition to other components of extracellular matrix (38), low levels of fibronectin in the germinal matrix might be attributed to diminished TGF $\beta$ expression of this periventricular brain region. Thus, up-regulation of TGF $\beta$ would enhance fibronectin level in the germinal matrix and might strengthen the frail vasculature of this brain area. However, TGF $\beta$ has diverse functions and its up-regulation to reduce the fragility of germinal matrix vasculature may not be a safe therapeutic strategy to prevent IVH.

Paucity of pericytes in the germinal matrix. Pericytes are cells of microvasculature-capillaries, venules, and arterioles-that wrap around the endothelial cells. They provide stability and structural integrity to the microvasculature (39). They are engaged in different stages of angiogenesis including initiation, sprout extension, migration, and maturation of blood vessels (40). With the initiation of angiogenesis, they degrade the basal lamina, migrate out of the blood vessels, and regulate endothelial proliferation and migration. Toward the end of angiogenesis, they resume their position and reinforce the vessel by synthesizing extracellular matrix and endothelial differentiation (41). At least four ligand-receptor system regulates pericyte recruitment and vessel maturation, the ligands being TGF- $\beta$, PDGF-B, angiopoietin, and sphingosine-1- 
phosphate1 (S1P1) (41). Mouse engineered to be deficient in any of the ligands or receptors develop endothelial tubes, but pericytes fail to associate with endothelial cells, resulting in irregular dilated vasculature with propensity to hemorrhage $(42,43)$.

Pericytes have been quantified in immunostained brain sections and electron micrographs of autopsy brain samples from fetuses and premature infants as well as in premature rabbit pups. Importantly, pericyte coverage and density are lesser in the germinal matrix vasculature than in the cortex or white matter in human fetuses, premature infants, and premature rabbit pups (Fig. 2B) (19). To determine the basis of fewer pericytes in the germinal matrix, the expression of four ligand-receptor system has been evaluated. The expression of PDGF-B and its receptor, PDGFR $\beta$, as well as angiopoietin-1 and its receptor Tie are comparable in the germinal matrix, cortex, and white matter (19). TGF- $\beta 1$ protein expression is lower, whereas S1P and N-cadherin levels are higher in the germinal matrix than in the cortex or white matter (19). TGF- $\beta 1$ contributes to stabilization of blood vessels by differentiation of pericytes from mesenchymal cells. Elevated S1P1 with N-cadherin assist vascular maturation. Low concentration of TGF- $\beta 1$ activates endothelial proliferation, whereas high dose of TGF- $\beta 1$ inhibits it (44). In conclusion, reduced density of pericytes in the germinal matrix vasculature may contribute to its propensity to hemorrhage, and low expression of TGF- $\beta 1$ could be a basis of paucity of pericytes in its vasculature.

Rapid angiogenesis in the germinal matrix vasculature. The germinal matrix exhibits rapid endothelial proliferation

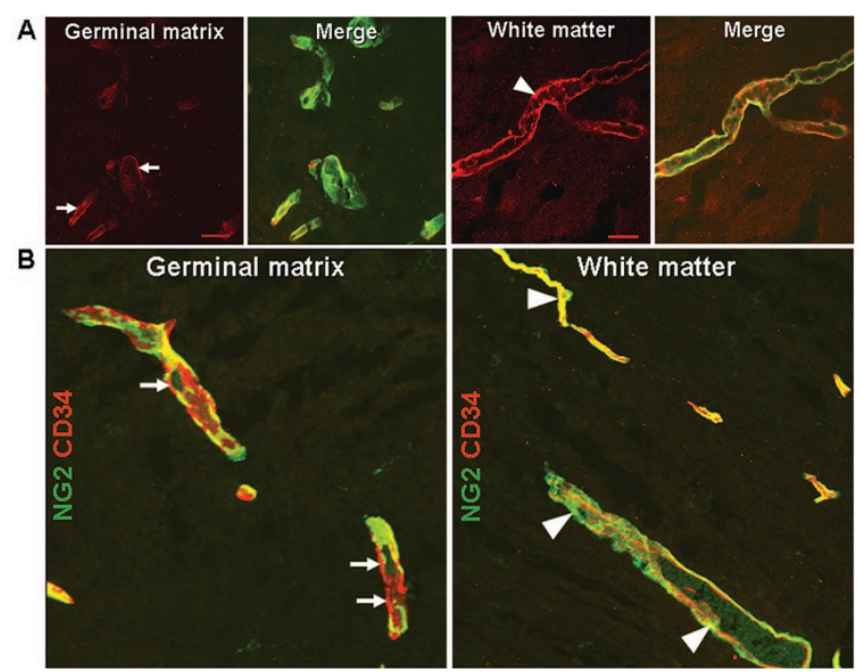

Figure 2. The germinal matrix is deficient in fibronectin and pericytes. (A) Representative immunofluorescence of cryosection from the germinal matrix and white matter of a $24-w k$ premature infant labeled with fibronectin (red) and CD34 (green)-specific antibody. Fibronectin is strongly expressed in the white matter (arrowheads), whereas it is weakly expressed in the germinal matrix (arrows). (B) Cryosection from the germinal matrix and white matter of a 24 -wk premature infant was double labeled with CD34 (endothelium, red) and NG2 (pericyte, green)-specific antibody. NG2-positive pericytes are intimately associated with the outer endothelial surface. The coverage of the endothelia by NG2-positive pericytes is interrupted and incomplete in the germinal matrix (arrow). In contrast, in the white matter, the vascular coverage by pericytes is continuous and complete (arrowhead). Scale bar; $20 \mu \mathrm{m}$. and accordingly, VEGF and angiopoietin-2 levels are higher in the germinal matrix than in the cerebral cortex or white mater in both humans and rabbits (Fig. 3A) (20). In addition, endothelial proliferation is greater in fetuses, who succumb within a few hours of birth, compared with premature infants of mean postnatal age of $7 \mathrm{~d}$ (20). This indicates that angiogenesis in the germinal matrix diminishes shortly after birth. More importantly, suppression of VEGF by prenatal celecoxib (a COX-2 inhibitor) or ZD6474 (VEGFR2 inhibitor) treatment suppresses the incidence of glycerol-induced IVH in premature rabbit pups (20). Treatment with these antiangiogenic agents suppresses endothelial proliferation, reduces vascular density, and increases pericyte coverage in the germinal matrix (19). This suggests that angiogenic inhibition results in pruning of nascent vessels deficient in pericytes. Thus, suppressing angiogenesis and trimming of germinal matrix vasculature can potentially result in hypoxia of the germinal matrix.

Importantly, the prevailing view was that antiangiogenic therapies destroy the vasculature, thereby depriving the tissue

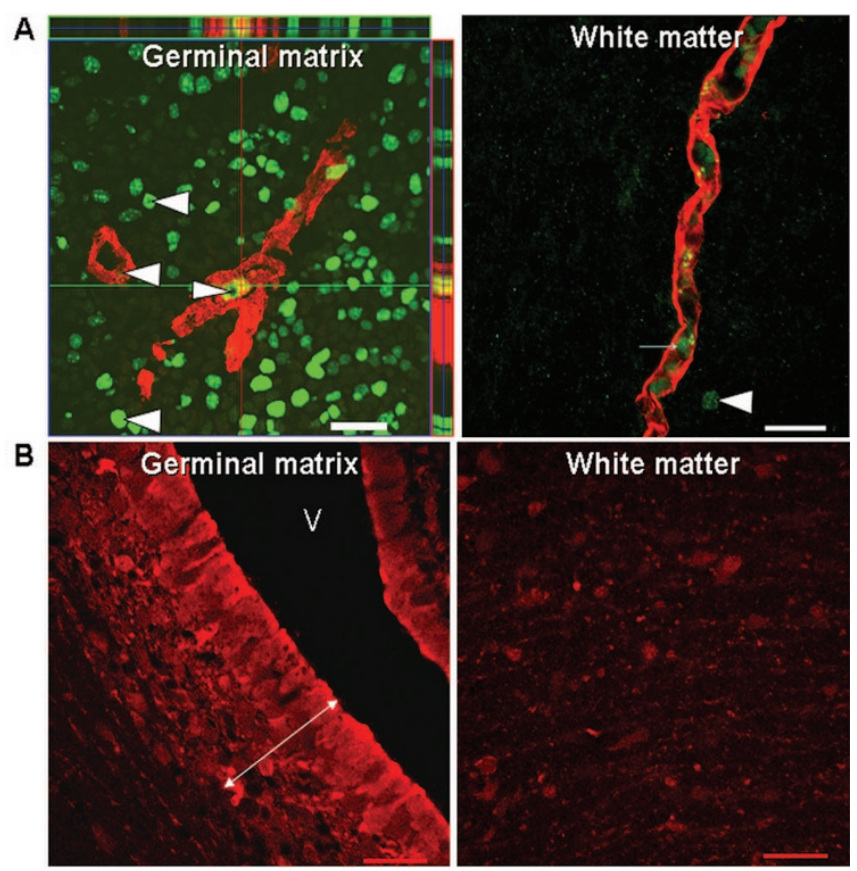

Figure 3. Rapid endothelial proliferation in the hypoxic germinal matrix. $(A)$ Representative immunofluorescence of cryosection from the germinal matrix and white matter of a 21-wk fetus labeled with Ki67 (proliferating nuclei, green) and CD34 (red)-specific antibody. Note: abundant Ki67-positive nuclei in the germinal matrix (arrowhead), but only few in the white matter. CD34 stained vessels with Ki67 signal (arrowhead) indicate endothelial proliferation in the germinal matrix. Left panel also shows orthogonal views in $\mathrm{x}-\mathrm{z}$ and $\mathrm{y}-\mathrm{z}$ planes of a composite stack of a series of images acquired by a confocal microscope. Right panel shows CD34-positive blood vessel with $\mathrm{RBC}$ in the lumen (arrow), but no Ki67-positive signal in the blood vessel. Scale bar; $20 \mu \mathrm{m}$. (B) A premature rabbit pup (E29) was injected with hypoxyprobe $(60 \mathrm{mg} / \mathrm{kg}$ dose $)$ immediately after birth and pup was euthanized after $2 \mathrm{~h}$. Hypoxyprobe is metabolized by nitroreductase enzymes in case of low $\mathrm{PO}_{2}$ level of to form hypoxyprobe chemical adducts. Frozen sections were stained with hypoxyprobe MAb to detect hypoxyprobe adducts. Note: intense immunostaining (double-headed arrow) in the periventricular germinal matrix and weak immunoreactivities in the white matter. V, ventricle. Scale bar; $20 \mu \mathrm{m}$. 
of oxygen and nutrients. However, recent evidence has shown that judicious use of certain angiogenic inhibitors normalize the abnormal structure and function of blood vessels in tumors, resulting in improved tissue oxygenation (45). During the normalization period, VEGFR2 blockade increases pericyte coverage by angiopoieitn-1 up-regulation and degrades pathologically thick basal lamina by matrix metalloproteinase activation $(46,47)$. On this basis, we speculate that titrating the dose of COX-2 inhibitor or VEGFR2 blocker might minimize pruning of germinal matrix vasculature and provide vascular stabilization by enhancing pericyte coverage and maturing basal lamina. Thus, prenatal antiangiogenic therapy in low dose for short period ( $2-3 \mathrm{~d}$ ) holds promise in the prevention of IVH and needs further preclinical testing to determine its safety and efficacy in suitable animal models of IVH.

Why a rapid endothelial turnover in the germinal matrix? The germinal matrix harbors neuronal and glial precursor cells, which are in different stages of proliferation, migration, and maturation. This indicates high metabolic demand and oxygen requirement of the germinal matrix. Notably, VEGF and ANGPT-2 are higher in the germinal matrix than in the cortex or white matter; and hypoxia is a key trigger of these growth factors (48). Therefore, it is tempting to hypothesize that the germinal matrix exhibits physiologic hypoxia. In a recent experiment, we used hypoxyprobe kit (Chemicon) to evaluate oxygen gradient between the germinal matrix and other brain areas in premature rabbit newborns. Hypoxyprobe is an exogenous nitroaromatic compound [1-[(2-hydroxy-3piperidinyl)propyl]-2-nitroimidazole hydrochloride] frequently used to study hypoxia in cancer. Hypoxyprobe is metabolized by cellular nitroreductase enzymes in case of low physiologic level of $\mathrm{PO}_{2}\left(\mathrm{Po}_{2}<10 \mathrm{~mm} \mathrm{Hg}\right)$. The resulting hypoxyprobe chemical adducts can be detected by specific antibody on immunohistochemistry of hypoxic tissues. Thus, premature rabbit pups (E29) were injected with hypoxyprobe immediately after birth and pups were killed after $2 \mathrm{~h}$. Frozen sections were labeled with hypoxyprobe MAb to detect hypoxyprobe adducts. We noted intense immunostaining in the germinal matrix and weaker immunoreactivities in the cortex, white matter, and other brain areas (Fig. $3 B$ ). The experiment suggests lower tissue oxygen level (relative hypoxia) in the germinal matrix compared with the other brain regions. However, the finding of physiologic hypoxia in the germinal matrix needs confirmation by using other methods such as oxygen microelectrode. Together, the germinal matrix might be hypoxic, resulting from rapid consumption of oxygen, which triggers production of VEGF and angiopoietin-2 (Fig. 4). Moreover, it is plausible that the decrease in VEGF, angiopoietin-2, and endothelial turnover as a function of postnatal age both in rabbits and humans results from increase in postnatal arterial oxygenation.

In conclusion, fragility of the germinal matrix vasculature can be attributed to a lack of pericytes and immaturity of basal lamina in the angiogenic vessels abundantly present in this germinal matrix. Rapid capillary remodeling in the germinal matrix vasculature can be ascribed to physiologic hypoxia distinctive of this brain region, which possibly results from its

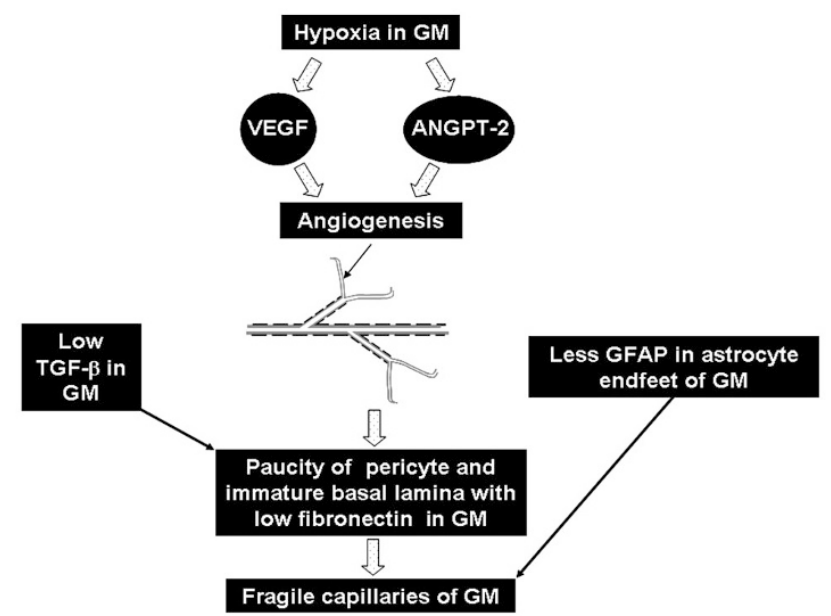

Figure 4. Mechanisms underlying fragility of the germinal matrix (GM) vasculature. The hypoxic germinal matrix induces VEGF and angiopoieitn-2 expression. These growth factors trigger angiogenesis. The nascent vessels of the germinal matrix exhibit paucity of pericytes and deficiency of fibronectin in immature basal lamina. In addition, astrocyte endfeet in the germinal matrix vasculature displays diminished expression of GFAP. These factors contribute to the fragility of the germinal matrix vasculature. Arrow in the schematic of neovasculature shows angiogenic blood vessel (gray, endothelium and black, pericyte) lacking pericytes.

high metabolic activity and oxygen consumption of its neural precursor cells.

\section{Disturbance in Cerebral Blood Flow}

Fluctuation in cerebral blood flow. Fluctuating CBF is associated with the development of IVH (49-51). Doppler technique has been used to measure CBF velocity in the premature infants with respiratory distress syndrome, who are on mechanical ventilator, on the first day of life. Two patterns of CBF velocity are delineated: a stable or a fluctuating CBF pattern. A stable CBF pattern consists of equal peak and trough of systolic and diastolic flow velocity, whereas a fluctuating CBF pattern comprises continuous alteration in systolic and diastolic blood flow velocity. The neonates with fluctuating CBF velocity have higher incidence of IVH compared with infants with stable CBF pattern (50). Similar observations were made by a subsequent investigator (52). Importantly, elimination of fluctuating $\mathrm{CBF}$ velocity by i.v. pancuronium substantially reduces the incidence of IVH (51). As paralysis removes infant's contribution to the ventilation and prevents asynchrony between infant and ventilator breath, there is diminution in $\mathrm{CBF}$ fluctuation resulting in restoration of a stable pattern. However, the routine use of neuromuscular blocking agent in ventilated newborn infants is not recommended because of an uncertainly regarding the long-term neurologic and pulmonary adverse effects of this medication. Notably, newer modes of ventilation, including synchronized intermittent mandatory ventilation and assist control are now widely practiced in modern NICUs worldwide, which synchronize patient and machine breathing and reduce the fluctuation in CBF velocity in infants (53). Other clinical conditions, including hypercarbia, hypotension, PDA, and 
restlessness, also contribute to the fluctuation in the CBF and have been correlated with the development of IVH $(52,54,55)$.

Pressure passivity of cerebral blood flow. Cerebral autoregulation is an ability of cerebral vessels to maintain a relatively constant $\mathrm{CBF}$ despite fluctuation in arterial blood pressure. The popular notion prevails that the sick premature infants are not able to sustain constant $\mathrm{CBF}$ at autoregulatory plateau, thus exhibiting pressure passivity of cerebral circulation. Cerebral autoregulation has been assessed by xenon clearance in earlier studies and more recently by Doppler, near-infrared spectroscopy (NIRS), or spatially resolved spectroscopy (SRS) (56-58). The pressure passivity of CBF directly correlates with lower gestational age and birth weight and is more frequently seen in sick, ventilated, and clinically unstable premature infants compared with clinically stable infants. In one of the earlier studies, the effect of acute changes in $\mathrm{CO}_{2}$ and blood pressure on global $\mathrm{CBF}$ was measured in premature infants on mechanical ventilator using xenon-133 clearance (59). It was noted that infants with pressure-passive circulation and reduced $\mathrm{CO}_{2}$ vasoreactivity developed IVH in contrast to infants with preserved autoregulation and $\mathrm{CO}_{2}$ vasoreactivity. In addition, global $\mathrm{CBF}$ was $20 \%$ less in infants who subsequently developed IVH compared with those who did not. Accordingly, evaluation of CBF by NIRS showed that the infants with impaired cerebral autoregulation were associated with a higher occurrence rate of IVH compared with neonates with effective autoregulation (60). Subsequent investigators realized the value of continuous recording of CBF for long periods using NIRS because cerebral autoregulation is not an "all-or-none" phenomena but a dynamic and evolving process (61). A successive larger study on VLBW infants (1500 g) showed that cerebral perfusion was pressure passive for an average of $20 \%$ of the time, whereas in some extremely low birth weight infant, this event exceeded $50 \%$ of the recording time (57). In this study, no relationship between occurrence of IVH and pressure passivity of cerebral circulation or systemic hypotension was observed (57). In another recent report, cerebral autoregulation was assessed by SRS, which is a recent development of NIRS and continuously measures cerebral oxygen saturation (tissue oxygen index). In this study, impaired autoregulation does not predict the development of IVH, but the pressure passivity of cerebral circulation in sick premature infants was associated with subsequent mortality (58). Thus, a static single evaluation of CBF by xenon clearance or NIRS may result in an erroneous interpretation, whereas continuous monitoring of $\mathrm{CBF}$ by NIRS or SDS is considered a preferred method to assess impaired autoregulation. Hence, impaired autoregulation and the subsequent development of IVH seem to be unrelated, but needs further confirmation.

Abnormal blood pressure, cerebral venous pressure, and other complications of prematurity. Hypotension is a diagnosis in $20-45 \%$ of premature infants, whereas hypertension is infrequent in these neonates $(62,63)$. A number of investigators have shown that systemic hypotension predisposes to IVH (64-66). Other investigators have, however, found no association between hypotension and IVH $(57,67,68)$. Elevations of cerebral venous pressure (CVP) have been implicated in contributing to the occurrence of IVH (69). Indeed, the germinal matrix hemorrhage has been demonstrated to be primarily venous in origin in an evaluation of autopsy materials from premature infants (70). Importantly, CVP is elevated in pneumothorax and during mechanical ventilation or any positive pressure ventilation; and high CVP might reduce the cerebral perfusion pressure (CPP). CPP is the difference between mean arterial pressure (MAP) and intracranial pressure (ICP) (CPP = MAP-ICP). However, when CVP is high with normal or low ICP, CPP $(\mathrm{CPP}=\mathrm{MAP}-\mathrm{CVP})$ is reduced resulting in a decrease in $\mathrm{CBF}$.

There is disagreement on the pathogenetic role of rapid infusion of sodium bicarbonate or hyperosmolar fluids in IVH $(71,72)$. These factors can potentially contribute to IVH by causing episodic increase in the $\mathrm{CBF}$. A rapid sodium bicarbonate infusion can also result in high arterial $\mathrm{CO}_{2}$ that can cause cerebral vasodilation. Sick premature infants in NICU undergo a number of events including hypercarbia, hypoxia, apnea, bradycardia, nonclosure of ductus arteriosus, requirement of high ventilator pressure, and others, which could result in fluctuation in blood pressure and alteration in cerebral hemodynamics. Determining accurately the effect of each of these events on $\mathrm{CBF}$ in the presence of several confounders and establishing a causal relationship of these clinical conditions with the development of IVH is quite challenging.

Regulation of cerebral blood flow: cellular and molecular mechanisms. Cerebral circulation is equipped with protective mechanisms to ensure adequate blood supply to the brain. One such mechanism is cerebral autoregulation and the other is functional hyperemia (73). Cerebral autoregulation, an inherent ability to maintain a relatively steady-state CBF despite fluctuation in arterial blood pressure, is attributed to an intrinsic ability of smooth muscle cells and pericytes to constrict or relax to minimize variation in $\mathrm{CBF}$ (myogenic response). The signaling event underlying myogenic response consists of an activation of stretch-activated $\mathrm{Ca}^{2+}$ channels by an increase in intravascular pressure (74). This results in an elevation in intracellular $\mathrm{Ca}^{2+}$ and subsequent stimulation of phospholipase A2, leading to the release of arachidonic acid from membrane phospholipids. Arachidonic acid metabolites, 20HETEs, inhibit $\mathrm{Ca}^{2+}$-dependent $\mathrm{K}+$ channels resulting in depolarization of smooth muscles and vasoconstriction.

Functional hyperemia implies an increase in CBF induced by neural activity to meet the local metabolic demand. This is a well-coordinated event involving neurons, astrocytes, and vascular cells. The interactions between these cells are not well understood. However, the prevailing view is that activation of glutamate receptors results in a postsynaptic increase in calcium, which stimulate enzymes that produce vasoactive agents (75). A number of vasoactive agents, including $\mathrm{CO}_{2}$, hydrogen ion, $\mathrm{K}^{+}$, adenosine, nitric oxide, arachidonic acid metabolites, and cytokines, have been linked with an increase in CBF (73). The vasodilatory effects of adenosine are mediated by cAMP through $\mathrm{K}_{\mathrm{ATP}}$ channels in vascular smooth muscles (76). Both nitric oxide and adenosine are key regulators of functional hyperemia (73). COX-2-derived prostanoids also enhance $\mathrm{CBF}$ and $\mathrm{COX}-1$ products have role in astrocyte-induced vasodilation $(77,78)$. Indeed, indometh- 
acin used for PDA closure in premature newborns reduces CBF (79).

\section{Disorders of Hemostasis: Platelet, Coagulation, and Cytokine Disturbances}

A number of studies have shown that thrombocytopenia is a risk factor for IVH $(80,81)$. The role of coagulopathy in pathogenesis of IVH has not been conclusively proved (82). Because inflammatory mediators and coagulation factors might contribute to the development of IVH, polymorphisms in TNF $\alpha$, IL-1 $\beta$, IL-4, IL-6, and IL-10 as well as mutations in coagulation proteins have been evaluated as probable genes to modify the risk and severity of IVH (83-85). There is preliminary evidence suggesting a role for cytokine genes as risk modifiers for IVH.

\section{CONCLUSION}

Despite major efforts made to elucidate the pathogenesis and prevent IVH in the last few decades, prevention of IVH remains an unsolved problem. Disturbance in CBF and fragility of the germinal matrix vasculature primarily contribute to the development of IVH. Hence, determining means either to effectively restore normal $\mathrm{CBF}$ without the use of harmful sedatives and paralytic agents or to enhance the stability of germinal matrix vasculature can potentially lead to a valid strategy in the prevention of IVH.

Acknowledgment. I thank Drs. Edmund F. LaGamma and Joseph Etlinger for their constant support and encouragement, and Joanne Abrahams for technical assistance with images.

\section{REFERENCES}

1. Guyer B, Hoyert DL, Martin JA, Ventura SJ, MacDorman MF, Strobino DM 1999 Annual summary of vital statistics-1998. Pediatrics 104:1229-1246

2. Heuchan AM, Evans N, Henderson Smart DJ, Simpson JM 2002 Perinatal risk factors for major intraventricular haemorrhage in the Australian and New Zealand Neonatal Network, 1995-97. Arch Dis Child Fetal Neonatal Ed 86:F86-F90

3. Philip AG, Allan WC, Tito AM, Wheeler LR 1989 Intraventricular hemorrhage in preterm infants: declining incidence in the 1980s. Pediatrics 84:797-801

4. Jain NJ, Kruse LK, Demissie K, Khandelwal M 2009 Impact of mode of delivery on neonatal complications: trends between 1997and 2005. J Matern Fetal Neonatal Med 22:491-500

5. Wilson-Costello D, Friedman H, Minich N, Fanaroff AA, Hack M 2005 Improved survival rates with increased neurodevelopmental disability for extremely low birth weight infants in the 1990s. Pediatrics 115:997-1003

6. Whitelaw A 2001 Intraventricular haemorrhage and posthaemorrhagic hydrocephalus: pathogenesis, prevention and future interventions. Semin Neonatol 6:135-146

7. Murphy BP, Inder TE, Rooks V, Taylor GA, Anderson NJ, Mogridge N, Horwood LJ, Volpe JJ 2002 Posthaemorrhagic ventricular dilatation in the premature infant: natural history and predictors of outcome. Arch Dis Child Fetal Neonatal Ed 87:F37-F41

8. Pinto-Martin JA, Whitaker AH, Feldman JF, Van Rossem R, Paneth N 1999 Relation of cranial ultrasound abnormalities in low-birthweight infants to motor or cognitive performance at ages 2, 6, and 9 years. Dev Med Child Neurol 41:826-833

9. Vohr BR, Allan WC, Westerveld M, Schneider KC, Katz KH, Makuch RW, Ment LR 2003 School-age outcomes of very low birth weight infants in the indomethacin intraventricular hemorrhage prevention trial. Pediatrics 111:e340-e346

10. Roze E, Van Braeckel KN, van der Veere CN, Maathuis CG, Martijn A, Bos AF 2009 Functional outcome at school age of preterm infants with periventricular hemorrhagic infarction. Pediatrics 123:1493-1500

11. Antoniuk S, da Silva RV 2000 Periventricular and intraventricular hemorrhage in the premature infants. Rev Neurol 31:238-243

12. Kenny JD, Garcia-Prats JA, Hilliard JL, Corbet AJ, Rudolph AJ 1978 Hypercarbia at birth: a possible role in the pathogenesis of intraventricular hemorrhage. Pediatrics 62:465-467

13. DiSalvo D 1998 The correlation between placental pathology and intraventricular hemorrhage in the preterm infant. The Developmental Epidemiology Network Investigators. Pediatr Res 43:15-19
14. Volpe JJ 1989 Intraventricular hemorrhage in the premature infant-current concepts. Part I. Ann Neurol 25:3-11

15. Ballabh P, Braun A, Nedergaard M 2004 Anatomic analysis of blood vessels in germinal matrix, cerebral cortex, and white matter in developing infants. Pediatr Res 56:117-124

16. Ballabh P, Hu F, Kumarasiri M, Braun A, Nedergaard M 2005 Development of tight junction molecules in blood vessels of germinal matrix, cerebral cortex, and white matter. Pediatr Res 58:791-798

17. El-Khoury N, Braun A, Hu F, Pandey M, Nedergaard M, Lagamma EF, Ballabh P 2006 Astrocyte end-feet in germinal matrix, cerebral cortex, and white matter in developing infants. Pediatr Res 59:673-679

18. Xu H, Hu F, Sado Y, Ninomiya Y, Borza DB, Ungvari Z, Lagamma EF, Csiszar A, Nedergaard M, Ballabh P 2008 Maturational changes in laminin, fibronectin, collagen IV, and perlecan in germinal matrix, cortex, and white matter and effect of betamethasone. J Neurosci Res 86:1482-1500

19. Braun A, Xu H, Hu F, Kocherlakota P, Siegel D, Chander P, Ungvari Z, Csiszar A, Nedergaard M, Ballabh P 2007 Paucity of pericytes in germinal matrix vasculature of premature infants. J Neurosci 27:12012-12024

20. Ballabh P, Xu H, Hu F, Braun A, Smith K, Rivera A, Lou N, Ungvari Z, Goldman SA, Csiszar A, Nedergaard M 2007 Angiogenic inhibition reduces germinal matrix hemorrhage. Nat Med 13:477-485

21. Hellstrom M, Gerhardt H, Kalen M, Li X, Eriksson U, Wolburg H, Betsholtz C 2001 Lack of pericytes leads to endothelial hyperplasia and abnormal vascular morphogenesis. J Cell Biol 153:543-553

22. Ballabh P, Braun A, Nedergaard M 2004 The blood-brain barrier: an overview: structure, regulation, and clinical implications. Neurobiol Dis 16:1-13

23. Wilkinson M, Hume R, Strange R, Bell JE 1990 Glial and neuronal differentiation in the human fetal brain 9-23 weeks of gestation. Neuropathol Appl Neurobiol 16:193-204

24. Gould SJ, Howard S 1987 An immunohistochemical study of the germinal layer in the late gestation human fetal brain. Neuropathol Appl Neurobiol 13:421-437

25. Sarnat HB 1992 Role of human fetal ependyma. Pediatr Neurol 8:163-178

26. Morrison RS, De Vellis J, Lee YL, Bradshaw RA, Eng LF 1985 Hormones and growth factors induce the synthesis of glial fibrillary acidic protein in rat brain astrocytes. J Neurosci Res 14:167-176

27. Hallmann R, Horn N, Selg M, Wendler O, Pausch F, Sorokin LM 2005 Expression and function of laminins in the embryonic and mature vasculature. Physiol Rev 85:979-1000

28. Tilling T, Engelbertz C, Decker S, Korte D, Huwel S, Galla HJ 2002 Expression and adhesive properties of basement membrane proteins in cerebral capillary endothelial cell cultures. Cell Tissue Res 310:19-29

29. George EL, Georges-Labouesse EN, Patel-King RS, Rayburn H, Hynes RO 1993 Defects in mesoderm, neural tube and vascular development in mouse embryos lacking fibronectin. Development 119:1079-1091

30. Forsberg E, Kjellen L 2001 Heparan sulfate: lessons from knockout mice. J Clin Invest 108:175-180

31. Gould DB, Phalan FC, Breedveld GJ, van Mil SE, Smith RS, Schimenti JC, Aguglia U, van der Knaap MS, Heutink P, John SW 2005 Mutations in Col4a1 cause perinatal cerebral hemorrhage and porencephaly. Science 308:1167-1171

32. Mao Y, Schwarzbauer JE 2005 Fibronectin fibrillogenesis, a cell-mediated matrix assembly process. Matrix Biol 24:389-399

33. Hocking DC, Sottile J, Langenbach KJ 2000 Stimulation of integrin-mediated cell contractility by fibronectin polymerization. J Biol Chem 275:10673-10682

34. Sottile J, Hocking DC, Langenbach KJ 2000 Fibronectin polymerization stimulates cell growth by RGD-dependent and -independent mechanisms. J Cell Sci 113:42874299

35. Ment LR, Stewart WB, Ardito TA, Madri JA 1991 Beagle pup germinal matrix maturation studies. Stroke 22:390-395

36. Ment LR, Stewart WB, Ardito TA, Huang E, Madri JA 1992 Indomethacin promotes germinal matrix microvessel maturation in the newborn beagle pup. Stroke 23:11321137

37. Anstrom JA, Thore CR, Moody DM, Challa VR, Block SM, Brown WR 2005 Morphometric assessment of collagen accumulation in germinal matrix vessels of premature human neonates. Neuropathol Appl Neurobiol 31:181-190

38. Pasinetti GM, Nichols NR, Tocco G, Morgan T, Laping N, Finch CE 1993 Transforming growth factor beta 1 and fibronectin messenger RNA in rat brain: responses to injury and cell-type localization. Neuroscience 54:893-907

39. Balabanov R, Dore-Duffy P 1998 Role of the CNS microvascular pericyte in the blood-brain barrier. J Neurosci Res 53:637-644

40. Hirschi KK, D'Amore PA 1997 Control of angiogenesis by the pericyte: molecular mechanisms and significance. EXS 79:419-428

41. Jain RK 2003 Molecular regulation of vessel maturation. Nat Med 9:685-693

42. Lindahl P, Johansson BR, Leveen P, Betsholtz C 1997 Pericyte loss and microaneurysm formation in PDGF-B-deficient mice. Science 277:242-245

43. Yancopoulos GD, Davis S, Gale NW, Rudge JS, Wiegand SJ, Holash J 2000 Vascular-specific growth factors and blood vessel formation. Nature 407:242-248

44. Bertolino P, Deckers M, Lebrin F, ten Dijke P 2005 Transforming growth factor-beta signal transduction in angiogenesis and vascular disorders. Chest 128:585S-590S

45. Jain RK 2005 Normalization of tumor vasculature: an emerging concept in antiangiogenic therapy. Science 307:58-62

46. Winkler F, Kozin SV, Tong RT, Chae SS, Booth MF, Garkavtsev I, Xu L, Hicklin DJ, Fukumura D, di Tomaso E, Munn LL, Jain RK 2004 Kinetics of vascular normalization by VEGFR2 blockade governs brain tumor response to radiation: role of oxygenation, angiopoietin-1, and matrix metalloproteinases. Cancer Cell 6:553563 
47. Uemura A, Ogawa M, Hirashima M, Fujiwara T, Koyama S, Takagi H, Honda Y, Wiegand SJ, Yancopoulos GD, Nishikawa S 2002 Recombinant angiopoietin-1 restores higher-order architecture of growing blood vessels in mice in the absence of mural cells. J Clin Invest 110:1619-1628

48. Mu D, Jiang X, Sheldon RA, Fox CK, Hamrick SE, Vexler ZS, Ferriero DM 2003 Regulation of hypoxia-inducible factor lalpha and induction of vascular endothelial growth factor in a rat neonatal stroke model. Neurobiol Dis 14:524-534

49. Tsuji M, duPlessis A, Taylor G, Crocker R, Volpe JJ 1998 Near infrared spectroscopy detects cerebral ischemia during hypotension in piglets. Pediatr Res 44:591595

50. Perlman JM, McMenamin JB, Volpe JJ 1983 Fluctuating cerebral blood-flow velocity in respiratory-distress syndrome. Relation to the development of intraventricular hemorrhage. N Engl J Med 309:204-209

51. Perlman JM, Goodman S, Kreusser KL, Volpe JJ 1985 Reduction in intraventricular hemorrhage by elimination of fluctuating cerebral blood-flow velocity in preterm infants with respiratory distress syndrome. N Engl J Med 312:1353-1357

52. Van Bel F, Van de Bor M, Stijnen T, Baan J, Ruys JH 1987 Aetiological role of cerebral blood-flow alterations in development and extension of peri-intraventricular haemorrhage. Dev Med Child Neurol 29:601-614

53. Rennie JM, South M, Morley CJ 1987 Cerebral blood flow velocity variability in infants receiving assisted ventilation. Arch Dis Child 62:1247-1251

54. Mullaart RA, Hopman JC, Rotteveel JJ, Daniels O, Stoelinga GB, De Haan AF 1994 Cerebral blood flow fluctuation in neonatal respiratory distress and periventricular haemorrhage. Early Hum Dev 37:179-185

55. Coughtrey H, Rennie JM, Evans DH 1997 Variability in cerebral blood flow velocity: observations over one minute in preterm babies. Early Hum Dev 47:63-70

56. du Plessis AJ 2008 Cerebrovascular injury in premature infants: current understanding and challenges for future prevention. Clin Perinatol 35:609-641

57. Soul JS, Hammer PE, Tsuji M, Saul JP, Bassan H, Limperopoulos C, Disalvo DN Moore M, Akins P, Ringer S, Volpe JJ, Trachtenberg F, du Plessis AJ 2007 Fluctuating pressure-passivity is common in the cerebral circulation of sick premature infants. Pediatr Res 61:467-473

58. Wong FY, Leung TS, Austin T, Wilkinson M, Meek JH, Wyatt JS, Walker AM 2008 Impaired autoregulation in preterm infants identified by using spatially resolved spectroscopy. Pediatrics 121:e604-e611

59. Pryds O, Greisen G, Lou H, Friis-Hansen B 1989 Heterogeneity of cerebral vasoreactivity in preterm infants supported by mechanical ventilation. J Pediatr 115:638-645

60. Tsuji M, Saul JP, du Plessis A, Eichenwald E, Sobh J, Crocker R, Volpe JJ 2000 Cerebral intravascular oxygenation correlates with mean arterial pressure in critically ill premature infants. Pediatrics 106:625-632

61. Reynolds KJ, Panerai RB, Kelsall AW, Rennie JM, Evans DH 1997 Spectral pattern of neonatal cerebral blood flow velocity: comparison with spectra from blood pressure and heart rate. Pediatr Res 41:276-284

62. Al-Aweel I, Pursley DM, Rubin LP, Shah B, Weisberger S, Richardson DK 2001 Variations in prevalence of hypotension, hypertension, and vasopressor use in NICUs. J Perinatol 21:272-278

63. Fanaroff JM, Fanaroff AA 2006 Blood pressure disorders in the neonate: hypotension and hypertension. Semin Fetal Neonatal Med 11:174-181

64. Miall-Allen VM, de Vries LS, Whitelaw AG 1987 Mean arterial blood pressure and neonatal cerebral lesions. Arch Dis Child 62:1068-1069

65. Watkins AM, West CR, Cooke RW 1989 Blood pressure and cerebral haemorrhage and ischaemia in very low birthweight infants. Early Hum Dev 19:103-110
66. Bada HS, Korones SB, Perry EH, Arheart KL, Ray JD, Pourcyrous M, Magill HL, Runyan W III, Somes GW, Clark FC 1990 Mean arterial blood pressure changes in premature infants and those at risk for intraventricular hemorrhage. J Pediat 117:607-614

67. Weindling AM, Wilkinson AR, Cook J, Calvert SA, Fok TF, Rochefort MJ 1985 Perinatal events which precede periventricular haemorrhage and leukomalacia in the newborn. Br J Obstet Gynaecol 92:1218-1223

68. Muller AM, Morales C, Briner J, Baenziger O, Duc G, Bucher HU 1997 Loss of CO reactivity of cerebral blood flow is associated with severe brain damage in mechanically ventilated very low birth weight infants. Eur J Paediatr Neurol 1:157-163

69. Volpe JJ 2008 Intracranial hemorrhage: germinal matrix hemorrhage. In: Volpe JJ (ed) Neurology of the Newborn. Saunders Elsevier, Philadelphia, pp 517-288

70. Ghazi-Birry HS, Brown WR, Moody DM, Challa VR, Block SM, Reboussin DM 1997 Human germinal matrix: venous origin of hemorrhage and vascular characteristics. AJNR Am J Neuroradiol 18:219-229

71. Wigglesworth JS, Keith IH, Girling DJ, Slade SA 1976 Hyaline membrane disease, alkali, and intraventricular haemorrhage. Arch Dis Child 51:755-762

72. Anderson JM, Bain AD, Brown JK, Cockburn F, Forfar JO, Machin GA, Turner TL 1976 Hyaline-membrane disease, alkaline buffer treatment, and cerebral intraventricular halphaemorrhage. Lancet 1:117-119

73. Iadecola C, Nedergaard M 2007 Glial regulation of the cerebral microvasculature. Nat Neurosci 10:1369-1376

74. Hill MA, Sun Z, Martinez-Lemus L, Meininger GA 2007 New technologies for dissecting the arteriolar myogenic response. Trends Pharmacol Sci 28:308-315

75. Iadecola C 2004 Neurovascular regulation in the normal brain and in Alzheimer's disease. Nat Rev Neurosci 5:347-360

76. Phillis JW 2004 Adenosine and adenine nucleotides as regulators of cerebral blood flow: roles of acidosis, cell swelling, and KATP channels. Crit Rev Neurobiol $16: 237-270$

77. Niwa K, Araki E, Morham SG, Ross ME, Iadecola C 2000 Cyclooxygenase-2 contributes to functional hyperemia in whisker-barrel cortex. J Neurosci 20:763-770

78. Niwa K, Haensel C, Ross ME, Iadecola C 2001 Cyclooxygenase-1 participates in selected vasodilator responses of the cerebral circulation. Circ Res 88:600-608

79. Upton RN, Rasmussen M, Grant C, Martinez AM, Cold GE, Ludbrook GL 2008 Pharmacokinetics and pharmacodynamics of indomethacin: effects on cerebral blood flow in anaesthetized sheep. Clin Exp Pharmacol Physiol 35:317-323

80. Andrew M, Castle V, Saigal S, Carter C, Kelton JG 1987 Clinical impact of neonatal thrombocytopenia. J Pediatr 110:457-464

81. Lupton BA, Hill A, Whitfield MF, Carter CJ, Wadsworth LD, Roland EH 1988 Reduced platelet count as a risk factor for intraventricular hemorrhage. Am J Dis Child 142:1222-1224

82. Shirahata A, Nakamura T, Shimono M, Kaneko M, Tanaka S 1990 Blood coagulation findings and the efficacy of factor XIII concentrate in premature infants with intracranial hemorrhages. Thromb Res 57:755-763

83. Gopel W, Hartel C, Ahrens P, Konig I, Kattner E, Kuhls E, Kuster H, Moller J, Muller D, Roth B, Segerer H, Wieg C, Herting E 2006 Interleukin-6-174-genotype, sepsis and cerebral injury in very low birth weight infants. Genes Immun 7:65-68

84. Harding DR, Dhamrait S, Whitelaw A, Humphries SE, Marlow N, Montgomery HE 2004 Does interleukin-6 genotype influence cerebral injury or developmental progress after preterm birth? Pediatrics 114:941-947

85. Harding D, Brull D, Humphries SE, Whitelaw A, Montgomery H, Marlow N 2005 Variation in the interleukin-6 gene is associated with impaired cognitive development in children born prematurely: a preliminary study. Pediatr Res 58:117-120 